\title{
Hepatocellular carcinoma - a challenge for oncologists
}

\author{
R. Anghel \\ Department of Radiotherapy, "Al. Trestioreanu" Institute of Oncology, University of Medicine and Pharmacy \\ "Carol Davilla" Bucharest, Bucharest, Romania
}

Received 15 June 2010, accepted 21 June 2010

Hepatocellular carcinoma (HCC) is one of the most common and lethal malignant disease and it represents a real challenge for nowadays oncology. In Europe we assist to an increase of both incidence and mortality of the disease. As European Cancer Observatory figures show, in 2006 the incidence of HCC in Europe was $12.1 \% 000$ inhabitants and the mortality was $10.1 \% 000$ inhabitants, increasing in the last years due to increasing incidence of infection with hepatitis $\mathrm{C}$ virus. The higher incidence was in Italy (22.0), France (18.9), and Greece (16.1) and the lowest in Malta (2.7), The Netherlands (2.7), and Norway (3.2). Regarding the mortality the first places in Europe are occupied by Italy (16.4), Romania (14.6), and France (14.4) and the last are Iceland (1.9), Norway (2.5), and The Netherlands (4.1). In Romania we had an incidence of $10.8 \% 000$ inhabitants (less than European media) and a mortality of $14.6 \% 000$ which is very high, placing us on the second place in Europe [1]. These figures justify the interest of Prof. Dr. Irinel Popescu for this topic. He is a well-known surgeon and a pioneer in liver transplant, being the one who realize the first liver transplant in Romania. His experience in the field is a very solid base for his article.

The incidence of HCC is mainly related to infection with hepatitis $\mathrm{B}, \mathrm{C}$ and $\mathrm{D}$ virus but there are some other etiologic factors like toxins (alcohol, tobacco and aflatoxins), hereditary metabolic liver disease, autoimmune hepatitis and newer factors like overweight in males, diabetes mellitus, and non-alcoholic fatty liver disease [2].

The prognosis of HCC is poor in principal because of detection in advanced stages when resection, the unique curative treatment, is not possible. For this reason more efforts should be done for early detection. The diagnostic is based on a combination of serum markers, imaging, and pathological assessment. So far the most frequently used serum marker is $\alpha$-fetoprotein. But it has relative low sensitivity and there is a need for more HCC-specific biomarkers. There are a series of such markers under research which seems to improve HCC diagnosis: Des- $\gamma$-carboxyprothrombin (DCP), $\alpha$-l-fucosidase (AFU), $\gamma$-glutamyl transferase (GGT), Glypcan-3 (GPC-3), Squamous cell carcinoma antigen (SCCA), etc. A new step for

Correspondence: Univ. Prof. Rodica Anghel, MD, Department of Radiotherapy, "Al. Trestioreanu" Institute of Oncology, University of Medicine and Pharmacy "Carol Davilla" Bucharest, 252 Fundeni Street, Sector 2, Bucharest, Romania.

E-mail: rodicamanghel@gmail.com
HCC testing is immunocomplexes of such markers with IgM immunoglobulins (AFP-IgM IC, SCCA-IgM IC and DCP-IgM IC). Unfortunately these serum biomarkers and their IgMimmunocomplexes still have significant diagnostic limitation and they are not very precise for the early diagnosis of HCC. Simultaneous determination of these markers in various combinations could improve the accuracy of detecting HCC. Gene expression profiling and microRNA assay will provide data about genomic alteration and could be a new tool to improve diagnostic and prognostic prediction of HCC [3]. According to Llovet, high accuracy rates are obtained by a 3-gene set: glypcan-3, LYVE1 (Lymphatic vessel endothelial hyaluronan receptor-1) and survivin [4].

The noninvasive imaging assessment is still not perfect for detecting HCC and for differentiating from other nodules, despite recent advances. Ultrasound is the most popular method for screening, with a sensitivity of $70 \%$ for lesions smaller than $1 \mathrm{~cm}$ rising to $90 \%$ for lesions greater than $5 \mathrm{~cm}$. The specificity varies from 48 to $94 \%$. Epidemiologic studies showed that only $50 \%$ of lesions smaller than $1 \mathrm{~cm}$ are detected by echography [4]. Spiral computed tomography (CT) and dynamic magnetic resonance imaging (MRI) are required for a better characterization of the tumour and for intrahepatic tumour staging. Dual-contrast-agent MRI administering SPIO (supermagnetic iron oxide) particles and gadolinium are examples of new contrast enhancers which can characterize liver nodules with greater confidence as mentioned in the article.

There are many systems which address the extent and the prognosis of the disease: Okuda staging system, TNM classification and its modification by UICC, Barcelona Clinic Liver Cancer (BCLC) classification, the Cancer of the Liver Italian Programme (CLIP) score, the Japan Integrated Staging (JIS) score, the Chinese University Prognostic Index (CUPI), etc. [2]. There is no consensus, but European Association for the Study of Liver Disease (EASLD) and American Association for the Study of Liver Diseases (AASLD) use BCLC as standard guidelines for clinical management of HCC.

Early stages HCC are potentially curable, but only $30-$ $40 \%$ of all patients are eligible for curative treatments. There are three main factors that can explain the therapeutic limits in HCC patients. First, HCC has an aggressive biologic behaviour which leads to both regional invasion and distant metastases at the time of diagnoses. Second, most cases of HCC appear on a chronic liver disease such as cirrhosis that can 
interfere with the curative treatments. Finally many cases are diagnosed in the advanced stages, which mean a lack of effective treatment options [5].

The therapeutic options are:

- Curative treatments: surgical resection, liver transplantation and in some situations loco-regional percutaneous interventions like radiofrequency thermal ablation therapy and percutaneous ethanol injection therapy.

- Non-curative (palliative) active treatments: transarterial intervention including trans-catheter arterial chemoembolization (TACE) and embolization, radiation therapy and systemic therapies like drugs as well as gene and immune therapies.

- Conservative management of symptoms or best supportive care.

Liver transplantation remains the definitive treatment for HCC complicating cirrhosis. Orthotopic liver transplant (OLT) was the universally accepted treatment for HCC since the Milan criteria were published. In the last years Living Donor Liver Transplant (LDLT) has evolved but it seems to have worse outcomes than OLT in HCC. The use of local ablative therapy in the period when waiting for transplantation appears to be accepted. Also resection as a bridge to transplantation seems to be a viable option for compensated patients. The use of neo-adjuvant drugs before surgery is also under investigation [6].

Radiotherapy has not frequently been used for treatment of HCC, considering the results of the early reports that radiation tolerance of liver was far less than the therapeutic radiation dose, leading to a low level of therapeutic ratio. Nowadays technical advance and a deeper understanding of radiobiology increase the use of radiotherapy in management of HCC. Radiotherapy has been proven not only for palliation but also for curative treatment for selected patients. Internal radiotherapy is performing by delivering radioisotopes either percutaneous or intraarterial; three types of isotopes were reported: Yttrium-90, Iodine-131 and Homium-199. External radiotherapy can be successfully performed for small tumours by using conformal techniques or SBRT (stereotactic body radiation therapy). It can also be used in combination with chemotherapy (mainly TACE). Particle beam radiotherapy like carbon ion beam treatment is under evaluation and is expected to improve treatment outcome in the future [7].

Until recently for patients with advanced stages no systemic therapy was available to improve survival, indicating the need for targeted-therapies. In 2007 Sorafenib was the first drug which showed overall survival benefit in patients with unresectable HCC. Beside this agent, several other molecules are currently tested.

The main molecular targets for treatment of HCC are:

- Ligand-membrane receptors signalling pathways like: EGFEGFR (epidermal growth factor receptor), Her2/neu, VEGF-
VEGFR (vascular endothelial growth factor receptor), PDGF-PDGFR (platelet-derived growth factor receptor), IGF-IGFR (insulin-like growth factor receptor), and HGFMET (hepatocyte growth factor - mesenchymal-epithelial transition factor).

- Intracellular signalling like: ras/MAPK (mitogen-activated protein kinase), PI3K/Akt/mTOR (phosphoinositide 3-kinase, mammalian target of rapamycin), Wnt/ $\beta$-catenin, and Hedgehog.

- RNA interference.

- Apoptosis.

The agents that are currently under development for HCC are: Gefitinib (EGFR targeting, phase 2 study), Erlotinib (EGFR targeting, phase 2 study), Lapatinib (EGFR targeting, phase 2 study), Cetuximab (EGFR targeting, phase 2 study), Bevacizumab (VEGF targeting, phase 1/2 study), Sunitinib (PDGFR, VEGFR, c-kit, FLT-3 targeting, phase 2 study), Vatalanib (VEGFR, PDGFR, c-kit targeting, phase 1 study), Cediranib (VEGFR targeting, phase 2 study), Rapamycin (mTOR targeting, phase 1/2 study), Everolimus (mTOR targeting, phase $1 / 2$ study), and Bortezomib (proteasome inhibitor, phase $1 / 2$ study) $[2,5]$.

Most probably, the combination of agents with different molecular targets like growth factor receptor inhibitors, proteasome inhibitors, or cytostatics will improve clinical outcome in patients with advanced HCC.

However, it should be noted that HCC is a complex disease with cancer and chronic liver disease. Therefore, achieving the therapeutic goals seems possible only through a multimodal team approach, which is a basic condition for good results.

The presented article can serve as a guide for current methods for diagnostic and treatment of HCC and also for future trends.

\section{Conflict of interest}

The author declares that there is no conflict of interest.

\section{References}

[1] European Cancer Observatory - http://eu-canceriarc.fr

2] Spangenberg HC, Thimme R, Blum HE. Evolving therapies in the treatment of hepatocellular carcinoma. Biologics Targets Ther, 2(3): 453-462, 2008

[3] Stefaniuk P, Cianciara J, Wiercinska-Drapalo A. Present and future possibilities for early diagnosis of hepatocellular carcinoma. World J Gastroenterol, 16(4): 418-424, 2010.

[4] Llovet JM, Chen Y, Wurmbach E, et al. A molecular signature to disriminate dysplastic nodules from early hepatocellular carcinoma in HCV cirrhosis. Gastroenterology, 131: 1758-1767, 2006.

[5] Song IH. Molecular targeting for treatment of advanced hepatocellular carcinoma. Korean J Hepatol, 15: 299-308, 2009.

[6] Tanwar S, Khan SA, Grover VPB, et al. Liver transplantation for hepatocellular carcinoma. World J Gastroenterol, 15(44): 5511-5516, 2009.

[7] Seong J. Challenge and hope in radiotherapy of hepatocellular carcinoma. Yonsei Med J, 50(5): 601-612, 2009. 